\title{
A New High-Intensity, Low-Momentum Muon Beam for the Generation of Low-Energy Muons at PSI
}

\author{
T. PROKSCHA*, E. MORENZONI, K. DEITERS, F. FOROUGHI, \\ D. GEORGE, R. KOBLER and V. VRANKOVIC \\ Paul Scherrer Institute, PSI, CH-5232, Villigen, Switzerland; e-mail: thomas.prokscha@psi.ch
}

\begin{abstract}
At the Paul Scherrer Institute (PSI, Villigen, Switzerland) a new high-intensity muon beam line with momentum $p<40 \mathrm{MeV} / \mathrm{c}$ is currently being commissioned. The beam line is especially designed to serve the needs of the low-energy, polarized positive muon source (LE- $\mu^{+}$) and LE- $\mu$ SR spectrometer at PSI. The beam line replaces the existing $\mu \mathrm{E} 4$ muon decay channel. A large acceptance is accomplished by installing two solenoidal magnetic lenses close to the muon production target $\mathrm{E}$ that is hit by the $590-\mathrm{MeV}$ PSI proton beam. The muons are then transported by standard large aperture quadrupoles and bending magnets to the experiment. Several slit systems and an electrostatic separator allow the control of beam shape, momentum spread, and to reduce the background due to beam positrons or electrons. Particle intensities of up to $3.5 \times 10^{8}$ $\mu^{+} / \mathrm{s}$ and $10^{7} \mu^{-} / \mathrm{s}$ are expected at $28 \mathrm{MeV} / \mathrm{c}$ beam momentum and $1.8 \mathrm{~mA}$ proton beam current. This will translate into a LE- $\mu^{+}$rate of $7,000 /$ s being available at the LE- $\mu$ SR spectrometer, thus achieving $\mu^{+}$fluxes, that are comparable to standard $\mu$ SR facilities.
\end{abstract}

Keywords: low-energy muons, muon beam.

At present the Paul Scherrer Institute (PSI, Villigen, Switzerland) is operating the most powerful proton accelerator at medium energies $(590 \mathrm{MeV})$ which is used for the generation of high-intensity, low-momentum muon beams at $100 \%$ duty cycle. These particles are used in stopped experiments which cover a large spectrum of physical problems, from fundamental particle physics experiments to condensed and soft matter investigations [1]. For the condensed and soft matter applications a large research program for muon-spin-rotation $(\mu \mathrm{SR})[2]$ has been established over the last 20 years. A new important extension of the $\mu$ SR program has been achieved by the recent development of a low-energy $\mu^{+}$beam (LE- $\mu^{+}$, 'LEM') with $\mu$ SR spectrometer (LE- $\mu$ SR) [3-6]. Whereas standard muon beams are produced with energies of order $\mathrm{MeV}$ the $\mathrm{LE}-\mu^{+}$beam has a tunable energy between 0.5 and $30 \mathrm{keV}$, thus allowing the controlled implantation at depths on the nanometer scale below the surface of a sample. The beam is generated by the moderation of a surface muon beam (momentum $p<29.8 \mathrm{MeV}$ ) in an appropriate solid rare gas film. Up to now LE- $\mu$ SR has been intensity limited. In order to use the full capacity of this new technique an increase of

\footnotetext{
*Author for correspondence.
} 


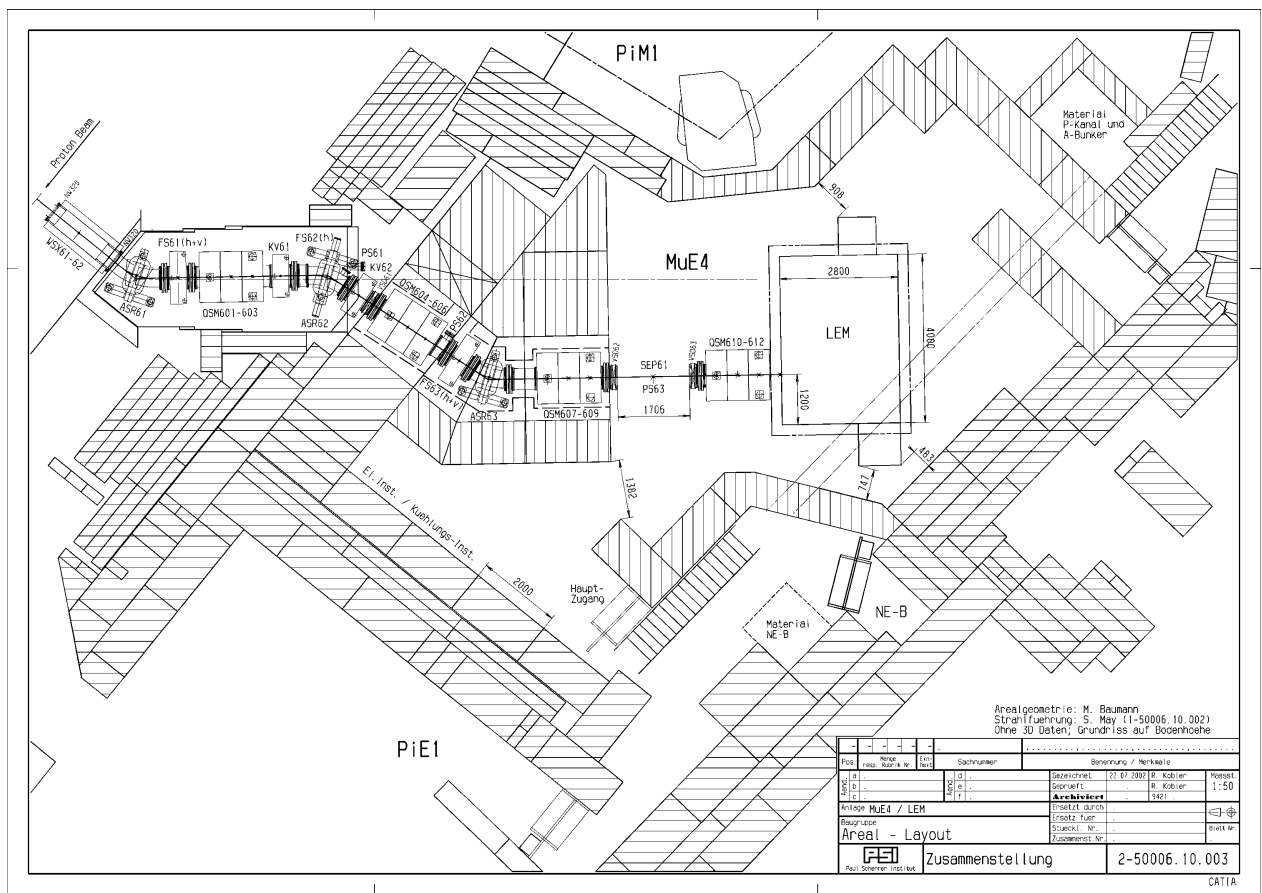

Figure 1. Layout of the new $\mu \mathrm{E} 4$ beam line and experimental area. LEM refers to the low-energy muon beam and LE- $\mu$ SR spectrometer.

muon beam intensity by about one order of magnitude at a beam momentum of $\sim 28 \mathrm{MeV} / \mathrm{c}$ is required. To achieve this purpose the existing $\mu \mathrm{E} 4$ channel at PSI has been completely redesigned to provide maximum $\mu^{+}$intensity at 28 $\mathrm{MeV} / \mathrm{c}$. The beam line can deliver $\mu^{-}$as well, with the maximum momentum restricted to $40 \mathrm{MeV} / \mathrm{c}$ due to limitations of the first focusing elements.

In order to increase significantly the acceptance of the beam line the existing standard quadrupoles had to be replaced by a solenoid as the first focusing element. The rotational symmetry of the solenoid allows to focus the beam in all directions. Disadvantages are the higher electric power consumption compared to a quadrupole lens with same focal length, and a mixing of the horizontal and vertical phase spaces. The new $\mu \mathrm{E} 4$ beam line uses two solenoids (WSX61, WSX62) where the first one can be operated at fields up to $0.37 \mathrm{~T}$, which limits the beam momentum to $40 \mathrm{MeV} / \mathrm{c}$. Both solenoids consist of normal conducting, radiation hard coils. By a proper combination of both elements a phase space rotation close to $90^{\circ}$ can be achieved, thus exchanging the initial horizontal and vertical phase space and keeping the mixing small. The solenoids are followed by large aperture $(240 \mathrm{~mm})$ bending magnets (ASR61-63) and quadrupole tripletts (QSM60l-612, aperture $400 \mathrm{~mm}$ ). A static $E \times B$ filter (SEP6I) with $180 \mathrm{~mm}$ electrode distance and up to $400 \mathrm{kV}$ 

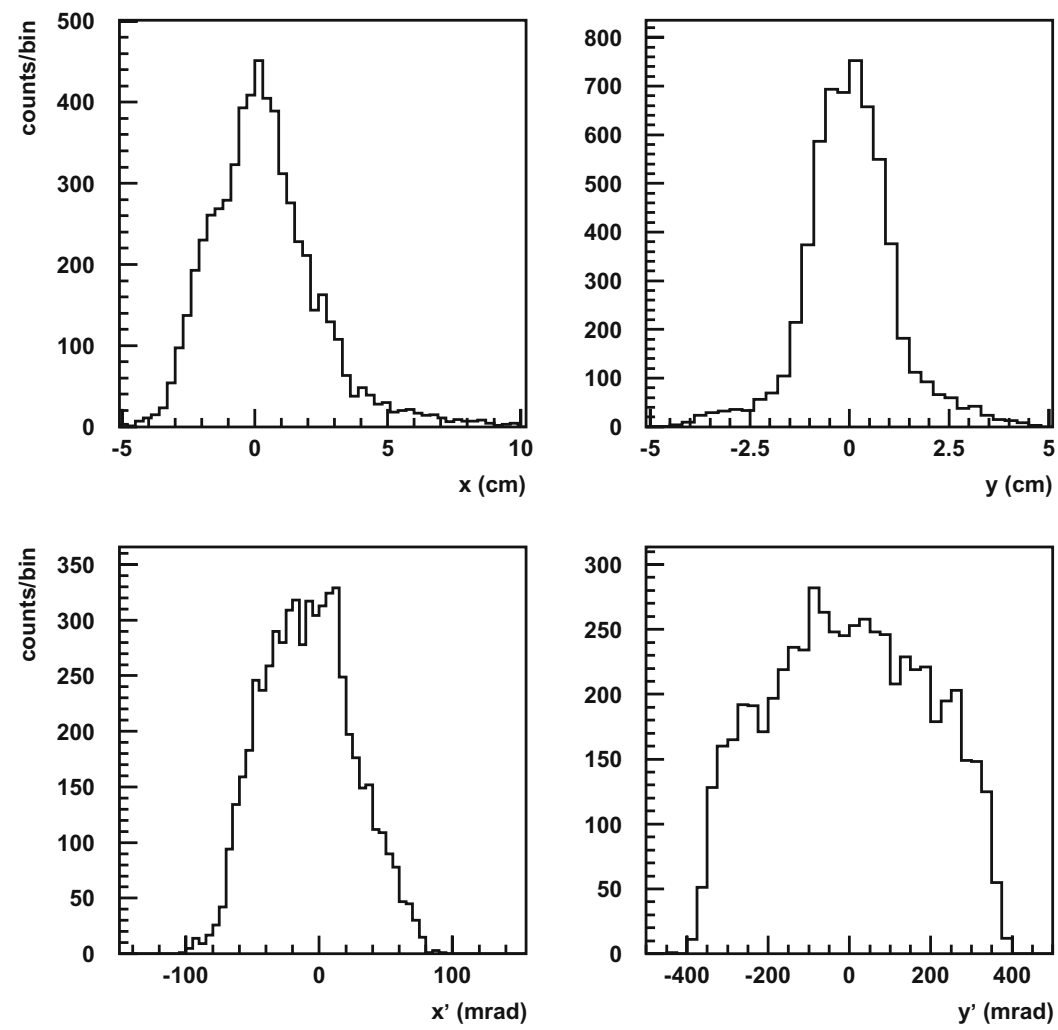

Figure 2. Phase space parameter of the new $\mu$ E4 beam line at the LEM moderator position as calculated by TURTLE [7]. $x$ and $y$ are the horizontal and vertical coordinates, respectively.

voltage difference will efficiently separate beam-contaminating positrons from the muons. Three-slit systems (FS61-63) allow the control of the beam intensity. The first and third device dispose of horizontal and vertical slits; the second one with horizontal slits is installed in the second bending magnet to adjust the beam momentum spread between $4.5 \%$ and $10 \%$ (FWHM). In a special operating mode, the beam line can achieve a momentum resolution of better than $2 \%$ at the expense of intensity. An overview of the new beam line with the area layout is shown in Figure 1.

Calculations of the beam line optics have been performed by the programs TRANSPORT and TURTLE [7] and by the ray-tracing program TRACK using calculated and measured field maps [8]. The calculated phase space parameters at the position of the LEM moderator are shown in Figure 2. A comparison with calculations for the existing $\pi$ E3 beam - where the LEM facility was running up to now - shows an anticipated increase of the LE- $\mu^{+}$intensity by a factor of 7 , see Table I. This will allow the exploitation of the full capacity of LE- $\mu \mathrm{SR}$. Routine operation is planned to start in 2005. 
Table I. Comparison of beam line parameters for the existing $\pi \mathrm{E} 3$ - that was used for LE- $\mu^{+}$ generation up to now - and the new $\mu \mathrm{E} 4$ channel

\begin{tabular}{lll}
\hline & $\pi \mathrm{E} 3$ achromatic mode & New $\mu \mathrm{E} 4$ \\
\hline Horizontal emittance & $60 \pi \mathrm{cm} \mathrm{mr}$ & $350 \pi \mathrm{cm} \mathrm{mr}$ \\
Vertical emittance & $24 \pi \mathrm{cm} \mathrm{mr}$ & $50 \pi \mathrm{cm} \mathrm{mr}$ \\
Accepted solid angle & $17 \mathrm{msr}$ & $135 \mathrm{msr}$ \\
$\Delta p / p(\mathrm{FWHM})$ & $7.5 \%$ & $4.5 \%-10 \%$ \\
On a $3 \times 3 \mathrm{~cm}^{2}$ area (LEM moderator) & $7.2 \%$ & $4.5 \%-7.5 \%$ \\
$\mu^{+}$intensity & $50 \times 10^{6} / \mathrm{s}$ & $350 \times 10^{6} / \mathrm{s}$ \\
$\mu^{+}$intensity on $3 \times 3 \mathrm{~cm}^{2}$ & $25 \times 10^{6} / \mathrm{s}$ & $180 \times 10^{6} / \mathrm{s}$ \\
Low-energy $\mu^{+}$rate (at moderator) & $3,000 / \mathrm{s}$ & $21,000 / \mathrm{s}$ \\
Low-energy $\mu^{+}$rate (at sample) & $1,000 / \mathrm{s}$ & $7,000 / \mathrm{s}$ \\
$x-y$ beam spot (FWHM) at end & $2.0 \times 3.0 \mathrm{~cm}^{2}$ & $4.0 \times 2.5 \mathrm{~cm}$ \\
$x-y$ beam divergence (FWHM) at end & $120 \times 50 \mathrm{mr}$ & $150 \times 450 \mathrm{mr}$ \\
Channel length & $15.5 \mathrm{~m}$ & $18.7 \mathrm{~m}$ \\
Positron contamination after separator & $2 \%$ & $2 \%$
\end{tabular}

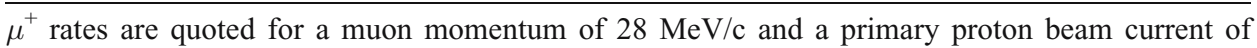
$1.8 \mathrm{~mA}$.

\section{Acknowledgements}

This work was performed at the Swiss Muon Source, Paul Scherrer Institute, Villigen, Switzerland. The construction of the beam line received important financial contributions from the German BMBF (TU Braunschweig and $U$ Konstanz), the UK EPSRC (U Birmingham), and from the Universities of Zürich and Leiden.

\section{References}

1. Petitjean C., In: Jacob M. and Schopper H. (eds.), Large Facilities in Physics, World Scientific, Singapore, 1995, p. 316.

2. Blundell S. J., Cont. Phys. 40 (1999), 175.

3. Morenzoni E. et al., Phys. Rev. Lett. 72 (1994), 2793.

4. Morenzoni E. et al., Physica B 289-290 (2000), 653.

5. Bakule P. and Morenzoni E., Cont. Phys. 45 (2004), 203.

6. Prokscha T., et al., these proceedings.

7. PSI Graphic TURTLE Framework by U. Rohrer, based on a CERN-SLAC-FERMILAB version by K.L. Brown et al.; PSI Graphic TRANSPORT Framework by U. Rohrer based on a CERN-SLAC-FERMILAB version by K.L. Brown et al.

8. TRACK: Three-dimensional Raytracing Analysis Computational Kit, developed by PSI Magnet section. 